\title{
Magnetic Properties of Ultrathin Magnetite Films Grown by Molecular Beam Epitaxy
}

\author{
S. K. Arora ${ }^{1}$, Han-Chun $\mathrm{Wu}^{1}$, Hongzhi Yao ${ }^{2}$, W. Y. Ching ${ }^{2}$, R. J. Choudhary ${ }^{3}$, I. V. Shvets ${ }^{1}$, and O. N. Mryasov ${ }^{4}$ \\ ${ }^{1}$ Centre for Research on Adaptive Nanostructures and Nanodevices (CRANN), School of Physics, Trinity College Dublin, \\ Dublin 2, Ireland \\ ${ }^{2}$ Department of Physics, University of Missouri-Kansas City, Kansas City, MO 64110 USA \\ ${ }^{3}$ UGC-DAE Consortium for Scientific Research, Indore, India \\ ${ }^{4}$ Seagate Research Center, Seagate Technology, Pittsburgh, PA 15222 USA
}

\begin{abstract}
Magnetic properties as a function of film thickness (2-55 nm) for magnetite $\left(\mathrm{Fe}_{3} \mathrm{O}_{4}\right)$ thin films grown on $\mathrm{MgO}(100)$ substrates show that the small thickness films $(\leq 5 \mathrm{~nm})$ are ferromagnetic and possess magnetization values which are much greater than that of bulk $\mathrm{Fe}_{3} \mathrm{O}_{4}$. The inverse thickness dependence of the magnetization points to an interface related mechanism. Whereas, our detailed spin polarized density functional theoretical calculations suggests that there is only a marginal enhancement in the local spin moments of Fe-atom in the vicinity of the $\mathrm{Fe}_{3} \mathrm{O}_{4}$-MgO interface and it turns out that the noncompensation of spin moments between the two magnetic sublattices of spinel $\mathrm{Fe}_{3} \mathrm{O}_{4}$ is a major factor contributing to the enhancement of magnetization.
\end{abstract}

Index Terms-Epitaxial layers, interface phenomena, magnetite, surfaces.

\section{INTRODUCTION}

$\mathbf{E}$ PITAXIAL heterostructures of half metallic oxides such as magnetite, $\mathrm{CrO}_{2}$ and rare Earth manganites have been the subject of great interest due to their potential applications in spintronics [1], [2]. Magnetite, $\mathrm{Fe}_{3} \mathrm{O}_{4}$, is an important half metallic oxide due to its high Curie temperature of $858 \mathrm{~K}$ and the presence of a metal-insulator transition at $120 \mathrm{~K}$ [3]. Various thin film growth techniques have been employed to grow epitaxial films of $\mathrm{Fe}_{3} \mathrm{O}_{4}$ on suitable substrates [4]-[8]. Transport and magnetic properties of epitaxial magnetite films have been investigated in detail [4]-[8].

Epitaxial $\mathrm{Fe}_{3} \mathrm{O}_{4}$ films grown on $\mathrm{MgO}$ substrate are reported to contain antiphase boundaries (APBs) [5]-[7]. Presence of these APB defects leads to the unusual magnetic properties of $\mathrm{Fe}_{3} \mathrm{O}_{4}$ films, in particular the nonsaturation of the magnetization, even at high field [5]-[7]. Voogt et al. [9] in their study on ultrathin epitaxial films $(<5 \mathrm{~nm})$ of $\mathrm{Fe}_{3} \mathrm{O}_{4}$ on $\mathrm{MgO}$ showed a superparamagnetic behavior of the films, which they attributed to the $180^{\circ} \mathrm{Fe}-\mathrm{O}-\mathrm{Fe}$ superexchange interaction resulting in a frustration of the interdomain interactions. In general, saturation is believed to be more difficult for thinner films as the density of the APBs is greater.

From these investigations, it is clear that the magnetization property of magnetite thin films immensely depend on the method of preparation, nature of defects and the defect density. Although, the presence of APBs leads to a deterioration of magnetic properties, their presence is beneficial in attaining a greater magnetoresistance response in epitaxial films than in the single crystals, provided these nano-scale defects are appropriately manipulated [7], [10].

Though the physical properties of $\mathrm{Fe}_{3} \mathrm{O}_{4} / \mathrm{MgO}$ films have been studied in detail, magnetic properties of ultrathin films remain a controversial topic. A recent report by Zhou et al. [11]

Digital Object Identifier 10.1109/TMAG.2008.2003173

Color versions of one or more of the figures in this paper are available online at http://ieeexplore.ieee.org. found that the ultrathin $\mathrm{Fe}_{3} \mathrm{O}_{4}$ films are ferromagnetic but their magnetization is reduced in comparison to the bulk values. They reported a magnetically inactive/dead layer of about $0.42 \mathrm{~nm}$. In these investigations, no emphasis was given to the surface and interface magnetism which is expected to strongly influence the magnetic properties. In fact, the theoretical predictions on a (100) $\mathrm{Fe}_{3} \mathrm{O}_{4}$ surface using first principal calculations suggest either an increase or decrease in the magnetic moment of the surface layer which strongly depends on the surface reconstruction [12], [19]. All above factors motivated us to carefully examine the magnetic response of the films at small thicknesses. In this paper, we report a systematic study of the magnetic properties of well characterized $\mathrm{Fe}_{3} \mathrm{O}_{4}$ films $(2-55 \mathrm{~nm})$. We show that the films are ferromagnetic in the smaller thickness $(<5 \mathrm{~nm})$ range and show a magnetic moment which is greater than the magnetic moment of bulk $\mathrm{Fe}_{3} \mathrm{O}_{4}$. We also compare the observed results with the theoretical calculations based on spin-polarized density functional theory for the $\mathrm{Fe}_{3} \mathrm{O}_{4}-\mathrm{MgO}$ (001) interface.

\section{EXPERIMENT}

Thin films of $\mathrm{Fe}_{3} \mathrm{O}_{4}$ were grown on (001) oriented $\mathrm{MgO}$ single crystal substrates using an oxygen plasma assisted molecular beam epitaxy (MBE) (DCA MBE M600, Finland) with a base pressure $5 \times 10^{-10}$ Torr. Substrate temperature during growth was $523 \mathrm{~K}$. Details of the growth procedure are given elsewhere [13]. The surface roughness of the substrates used in this study was $<0.4 \mathrm{~nm}$ as determined from the $5 \mu \mathrm{m} \times 5 \mu \mathrm{m}$ area scans using an atomic force microscope, AFM, (Solver Pro, NT-MDT, Russia). After completion of growth, the films were cooled to room temperature $(300 \mathrm{~K})$ with a cooling rate of $10 \mathrm{~K} / \mathrm{min}$. Reflection high energy electron diffraction (RHEED) was employed to confirm the epitaxial growth and establish the growth mode. The presence of the RHEED intensity oscillations confirms that the films grew in a layer-by-layer mode with a growth rate of $0.3 \AA$ As.

The single phase structural and epitaxial nature of the $\mathrm{Fe}_{3} \mathrm{O}_{4}$ films was characterized using a multi-crystal high-resolution X-ray diffractometer, HRXRD (Bede-D1, Bede, U.K.). Monochromatic $\mathrm{Cu}_{\mathrm{k} \alpha 1}(1.54056 \AA)$ radiation with a 20 arcsec beam divergence was obtained using four $\mathrm{Si}$ channel-cut 
crystals. X-ray reflectivity investigations in these films revealed a small interface $(0.3-0.5 \mathrm{~nm})$ roughness between the $\mathrm{Fe}_{3} \mathrm{O}_{4}-\mathrm{MgO}$ interface. The surface roughness of these films was quite small $(0.2-0.4 \mathrm{~nm})$ as estimated from $5 \mu \mathrm{m} \times 5 \mu \mathrm{m}$ area scans using an AFM. Magnetic properties of the films were examined by performing magnetization measurements using a vibrating sample magnetometer (Quantum Design-14 T Physical Property Measurements System) with a sensitivity of $5 \times 10^{-7} \mathrm{emu}$. The magnetization verses field (M-H) loops were measured by applying the magnetic field (maximum field of 1 Tesla) in the film plane along the (001) direction. The diamagnetic contribution from the sample holder and $\mathrm{MgO}$ substrate was obtained from independent $\mathrm{M}-\mathrm{H}$ loops and subtracted from the film's data. The uncertainty in measuring the absolute value of magnetization for the films is about $3 \%$.

\section{RESULTS AND DISCUSSION}

Prior to discussing the magnetization results we would like to mention that through detailed structural characterization using high-resolution X-ray diffraction, we have found that the $\mathrm{Fe}_{3} \mathrm{O}_{4}$ films maintain a one-to-one registry with the substrate. The in-plane and out-of-plane lattice parameter for the films as determined from the asymmetric (311) and symmetric (200) diffraction scans are $0.84266 \pm 0.00005 \mathrm{~nm}$ (twice of the $\mathrm{MgO}$ lattice constant) and $0.83717 \pm 0.00005 \mathrm{~nm}$, respectively. Details of the structural characterization were published in an earlier report [13]. The unit cell volume, which is consistent with bulk magnetite, indicates that the films are stoichiometric. No evidence of the presence of any other iron oxide phase or unreacted $\mathrm{Fe}$ was found from our high resolution XRD and TEM studies [13]. In addition to the structural investigations, we also measured the Verwey transition temperature $\left(T_{v}\right)$ in these films through magnetic measurements. All the films down to $2 \mathrm{~nm}$ thickness exhibited a $\mathrm{T}_{\mathrm{v}}$. Its value being 118 and $85 \mathrm{~K}$ for the 50 and $2 \mathrm{~nm}$ films, respectively. Presence of $\mathrm{T}_{\mathrm{v}}$ in our films indicate that the stoichiometry of the films is quite close to $\mathrm{Fe}_{3} \mathrm{O}_{4}$.

Fig. 1 shows the magnetic hysteresis loops (HL) of $\mathrm{Fe}_{3} \mathrm{O}_{4}$ films $(5,15 \mathrm{~nm}$ thickness $)$ measured at $300 \mathrm{~K}$ with an applied in-plane magnetic field. The $15 \mathrm{~nm} \mathrm{Fe}_{3} \mathrm{O}_{4}$ films show a saturation behavior with a moderate magnetic field $(8 \mathrm{kOe})$. For the $5 \mathrm{~nm}$ film, at large magnetic fields, the magnetization (M) exhibited a finite slope with the magnetic field. The magnetization values for the 5 and $15 \mathrm{~nm} \mathrm{Fe}_{3} \mathrm{O}_{4}$ films are found to be 914 and $615 \mathrm{emu} / \mathrm{cm}^{3}$, respectively. The coercivity $\left(H_{c}\right)$ values for the same films are 70 and 220 Oe, respectively. For film thickness $20 \mathrm{~nm}$ or greater, we find that the magnetization values are quite close to the values for bulk $\mathrm{Fe}_{3} \mathrm{O}_{4}\left(509 \mathrm{emu} / \mathrm{cm}^{3}\right.$ for $55 \mathrm{~nm}$ film $)$. In Fig. 2 we show the $M(10 \mathrm{kOe})$ value measured at $300 \mathrm{~K}$ for different thickness $\mathrm{Fe}_{3} \mathrm{O}_{4}$ films. One can notice that the magnetization of the films increases substantially with the decrease in films thickness. Magnetization values for the thin films are significantly larger than that of bulk $\mathrm{Fe}_{3} \mathrm{O}_{4}$ (4 $\mu_{\mathrm{B}} /$ f.u.). For example, for a 5-nm film, the magnetization is found to be $914 \mathrm{emu} / \mathrm{cm}^{3}$ (7.7 $\mu_{\mathrm{B}} / \mathrm{f} . \mathrm{u}$.) at $10 \mathrm{kOe}$ field. This behavior was repeatedly observed for the small thickness films $(2-15 \mathrm{~nm})$. As the film thickness increases, the magnetization decreases. This is a curious observation in a sense that much of the earlier published work related to ultra thin magnetite films suggested either the formation of a magnetically dead layer or

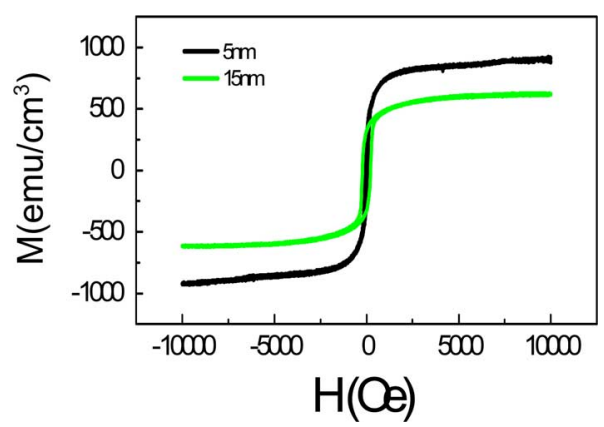

Fig. 1. Magnetization hysteresis (M-H) loops of $\mathrm{Fe}_{3} \mathrm{O}_{4}$ films measured at 300 $\mathrm{K}$ with an in-plane magnetic field.

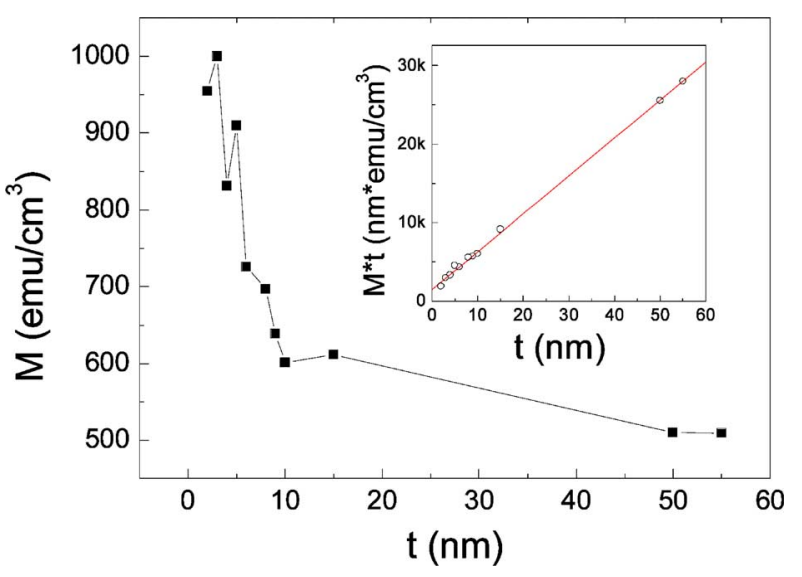

Fig. 2. Magnetization (M) measured at $300 \mathrm{~K}$ and $1 \mathrm{~T}$ filed as a function of film thickness. The inset shows M.t versus film thickness for $\mathrm{Fe}_{3} \mathrm{O}_{4}$ films.

the presence of superparamagnetism [9], [11]. In these earlier investigations, reduction in the magnetization was ascribed to the frustrated exchange at the APBs. We find that all the films down to $2 \mathrm{~nm}$ thickness exhibit a ferromagnetic behavior. Observation of a substantial (Giant) increase (95\%) in magnetization for a 5-nm $\mathrm{Fe}_{3} \mathrm{O}_{4}$ films is in stark contrast to the earlier notion of a reduction in magnetization or a superparamagnetic behavior [5], [9], [11]. Lower surface roughness of the substrates is a key to the nonobservation of superparamagnetism as opposed to previous reports. We have found that the films grown on higher surface roughness than $0.4 \mathrm{~nm}$ leads to a greater surface and interface roughness which leads to a reduced magnetization or superparamgnetism depending on the film thickness. For 2 and $4 \mathrm{~nm}$ films, we find a slightly reduced magnetization as compared to 3 and $5 \mathrm{~nm}$ films, respectively, which can be attributed to the partial noncompensation of the spin moment of two magnetic sublattice of spinel $\mathrm{Fe}_{3} \mathrm{O}_{4}$ arising due to a small uncertainity in the films thickness $(<0.03 \mathrm{~nm})$.

In order to understand the origin of the enhanced magnetization, we modeled our $\mathrm{Fe}_{3} \mathrm{O}_{4}$ films as consisting of a bulk-like $\mathrm{Fe}_{3} \mathrm{O}_{4}$ layer with a bulk saturation magnetization, $M_{s}$, and other thickness dependent contributions, $M_{i}$, (which are related to the interface, surface, and other contributions), then the magnetization $M$ is given by

$$
M=M_{s}+\frac{M_{i}}{t}
$$

where $\boldsymbol{t}$ is the thickness of the film. The inset of Fig. 2, shows the thickness dependence of $\mathrm{M}(10 \mathrm{kOe})$ multiplied 
by the $\mathrm{Fe}_{3} \mathrm{O}_{4}$ film thickness. Slope of the fit using (1) is $483 \mathrm{emu} / \mathrm{cm}^{3}$ and agrees with the value of magnetic moment of bulk $\mathrm{Fe}_{3} \mathrm{O}_{4}\left(480 \mathrm{emu} / \mathrm{cm}^{3}\right)$. The positive intercept on the $y$ axis $\left(1483 \mathrm{~nm} . \mathrm{emu} / \mathrm{cm}^{3}\right)$ is the magnetic contribution to the film arising from thickness dependent contributions. Our data suggests that the earlier proposition of a dead layer model for the $\mathrm{Fe}_{3} \mathrm{O}_{4} / \mathrm{MgO}$ interface was an oversimplification [11]. The inverse thickness dependence of effective magnetization (M) given by the model (1) to separate out distinct bulk $M_{s}$ and interface contributions is questionable, in particular for the case of $\mathrm{Fe}_{3} \mathrm{O}_{4}$ films on $\mathrm{MgO}$ (100) [11]. Apart from the interfaceand surface-related contribution for ultrathin films, an additional thickness dependent magnetization contribution could result from a totally different origin, i.e., from the thickness dependence of antiphase domain size.

In order to gain additional insights into surface/interface related contributions to the magnetization, we carried out a spinpolarized density functional theoretical calculations for a series of $\mathrm{Fe}_{3} \mathrm{O}_{4}-\mathrm{MgO}(001)$ interfaces with different slab thicknesses based on either $A$ (tetrahedral)- or $B$ (octahedral)-layer nucleation and their relative positions on the $\mathrm{MgO}$ (001) surface and compare them with those for bulk $\mathrm{Fe}_{3} \mathrm{O}_{4}$. The interface model was constructed according to the stacking sequence shown in Fig. 3 for the [001] direction. All calculations were performed using the VASP code with the projector augmented plane wave (PAW) method using generalized gradient approximation (GGA) [14]. We use $300 \mathrm{eV}$ for the plane wave energy cutoff, and $6 \times 6 \times 2$ grid for $\mathbf{k}$-point integration. All structural relaxation calculations are performed until the forces on the atoms become less than $20 \mathrm{meV} / \mathrm{atom}$. From the calculations for $\mathrm{Fe}_{3} \mathrm{O}_{4}-\mathrm{MgO}$ interface, we infer that the interface of $\mathrm{Fe}_{3} \mathrm{O}_{4}$ with $B$-site nucleation is energetically most stable. We performed a detailed band structure calculation for three different slab thicknesses corresponding to 1, 1.5, and 2 unit cell thick $\mathrm{Fe}_{3} \mathrm{O}_{4}$ layers on $\mathrm{MgO}$. Fig. 4 illustrates the layer structure used for Model-1-1. $\mathrm{Fe}-\mathrm{O}$ layer in the structure used for the calculations $\mathrm{Fe}$-O layers are the $\mathrm{Fe}_{\mathrm{B}}$-type layer as shown in Fig. 4. The model-1-1 consist of $5 \mathrm{Fe}_{\mathrm{B}}$-type, $4 \mathrm{Fe}_{\mathrm{A}}$-type layers and $5 \mathrm{MgO}$ layers. The slab is surrounded by a $1-\mathrm{nm}$ vacuum layer on either side of the slab. Details of the parameters used in the calculation and results are summarized in Table I. The magnetic moments of $\mathrm{Fe}$ atoms in $\mathrm{Fe}_{\mathrm{B}}$ layer in the vicinity of $\mathrm{Fe}_{3} \mathrm{O}_{4}-\mathrm{MgO}$ interface are comparable to that obtained for bulk $\mathrm{Fe}_{3} \mathrm{O}_{4}$ from a similar calculation. However, an increase in polarization of some of the oxygen atoms (as large as $0.36 \mu \mathrm{B}$ ) in the vicinity of interface was noted. These calculations suggest that the spin moment contribution from the $\mathrm{Fe}_{3} \mathrm{O}_{4}-\mathrm{MgO}$ interface is not enough to explain our observation of an enhanced magnetization. It has been shown that inclusion of electron correlations via on-site Coulomb interaction (U) in DFT calculation is important to understand magnetic properties and charge ordering phenomena in magnetite [15], [16]. A recent GGA +U study [17] suggest that the electron correlation effects influence the magnetization and shows an enhanced magnetization (by a few percent) for B-terminated $\mathrm{Fe}_{3} \mathrm{O}_{4}(100)$ surface. It is clear from the above discussion that the inclusion of the electronic correlation effects will not influence the results of DFT calculation presented in this study.

To explore the mechanism of magnetization enhancement in $\mathrm{Fe}_{3} \mathrm{O}_{4}$ films, we consider a noncompensated structure, i.e., one

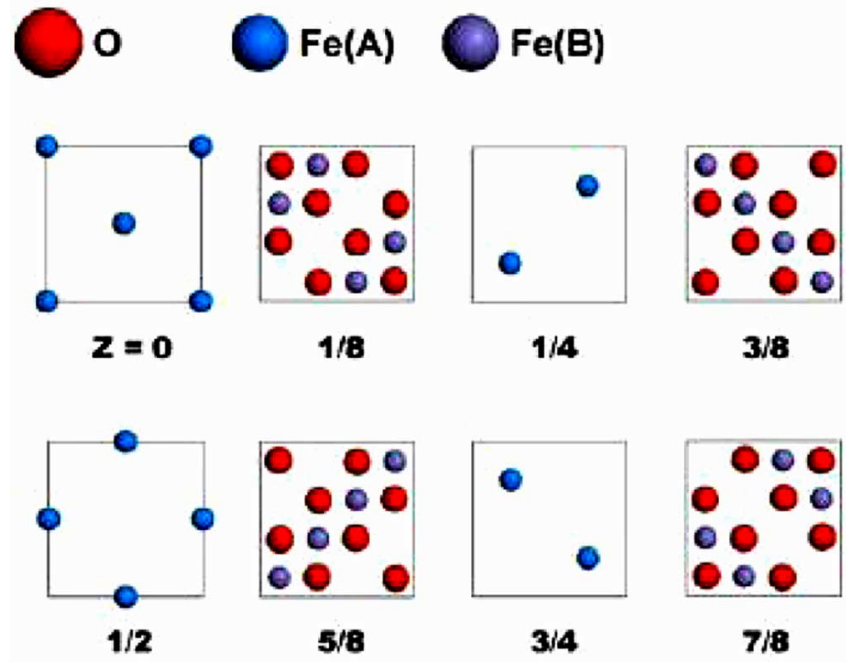

Fig. 3. Schematic illustration of the layered structure of $\mathrm{Fe}_{3} \mathrm{O}_{4}$ along the $\mathrm{Z}$ direction used for the DFT calculations, where the layers with $\mathrm{z}=0,1 / 4,1 / 2,3 / 4$ represent $\mathrm{A}$-termination and the layers with $\mathrm{z}=1 / 8,3 / 8,5 / 8,7 / 8$ represent $\mathrm{B}$-termination of $\mathrm{Fe}_{3} \mathrm{O}_{4}$ (001) surface.

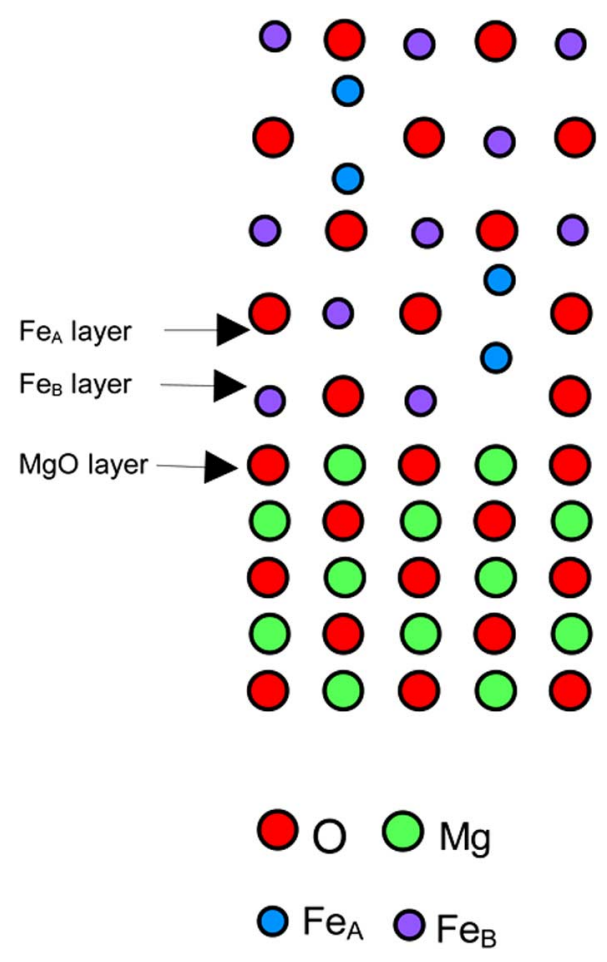

Fig. 4. Schematic illustration of the Model-1-1 used for the DFT simulations. The model utilizes $5-\mathrm{Fe}_{\mathrm{B}}$ type, $4 \mathrm{Fe}_{\mathrm{A}}$ type and $5 \mathrm{MgO}$ layers. The $\mathrm{Mg}$ atoms are represented with the green spheres.

with an odd number of $\mathrm{Fe}-\mathrm{O}$ planes. If a unit cell is not complete, one expects an increase in the magnetic moment due to noncancelation of the magnetic moments between $A$ - and $B$-site $\mathrm{Fe}^{3+}$ ions. We call this mechanism a noncompensation effect. It is clear that the noncompensation of spin moments due to an odd number of layers will enhance the magnetization of the surface layers and its effect will vanish for larger thickness samples. To estimate the effect of noncompensation we used a supercell 
TABLE I

Summary OF LATTICED PARAMETER, Film THICKNESS SPIN POLARIZATION, Total Magnetic Moments of the Cell, and Average Magnetic MOMENTS OF $\mathrm{FE}_{\mathrm{A}}$ AND $\mathrm{FE}_{\mathrm{B}}$ ATOMS FOR $\mathrm{FE}_{3} \mathrm{O}_{4}$ CRYSTAL AND OUR LOWEST ENERGY $\mathrm{FE}_{3} \mathrm{O}_{4}(001) / \mathrm{MGO}(001)$ INTERFACE MODEL WITH DIFFERENT THICKNESS OF $\mathrm{FE}_{3} \mathrm{O}_{4}$ FILMS. NUMERAL IN THE END OF MODEL'S NAME INDICATES THE THICKNESS OF $\mathrm{FE}_{3} \mathrm{O}_{4}^{4}$ LAYER IN UNITS OF UNIT CELL SIZE

\begin{tabular}{|c|c|c|c|c|}
\hline Results Model & Bulk & Model-1-1 & Model-1-1.5 & Model-1-2 \\
\hline \# of Atoms & 56 & 74 & 104 & 134 \\
\hline \# of $\mathrm{Fe}_{\mathrm{A}}$ & 8 & 4 & 6 & 8 \\
\hline \# of $\mathrm{Fe}_{\mathrm{B}}$ & 16 & 10 & 14 & 18 \\
\hline Film Thickness (nm) & -- & 0.8394 & 1.2591 & 1.6788 \\
\hline$a(\mathrm{~nm})$ & 0.8394 & 0.59529 & 0.59529 & 0.59529 \\
\hline $\mathbf{b}(\mathbf{n m})$ & 0.8394 & 0.59529 & 0.59529 & 0.59529 \\
\hline $\mathbf{c}(\mathbf{n m})$ & 0.8394 & 2.10275 & 2.93803 & 3.77993 \\
\hline Spin-polarization & 1 & 0.816 & 0.906 & 0.931 \\
\hline Total $M\left(\mu_{B}\right)$ & 31.591 & 27.089 & 34.993 & 42.834 \\
\hline Average $\mathrm{M}_{\mathrm{FeA}}\left(\mu_{\mathrm{B}}\right)$ & -3.430 & -3.402 & -3.413 & -3.420 \\
\hline Average $M_{\text {FeB }}\left(\mu_{B}\right)$ & 3.531 & 3.410 & 3.667 & 3.632 \\
\hline
\end{tabular}

model with a slab thickness equivalent to $50 \mathrm{Fe}-\mathrm{O}$ layers and find that the spin moment/f.u. is substantially larger due to noncompensated surface layer; e.g., its value being $6.6 \mu \mathrm{B} / \mathrm{f}$.u. for the fifth $\mathrm{Fe}-\mathrm{O}$ layer and $5 \mu \mathrm{B} / \mathrm{f}$.u. for fifteenth $\mathrm{Fe}-\mathrm{O}$ layer. The value of magnetizations decreases rapidly for a thicker slab and for a fiftieth $\mathrm{Fe}-\mathrm{O}$ layer, we find that the magnetization is comparable to the value for bulk $\mathrm{Fe}_{3} \mathrm{O}_{4}$, i.e., $\sim 4 \mu \mathrm{B} /$ f.u. For an even number of layers or fully compensated $\mathrm{Fe}_{3} \mathrm{O}_{4}$ slabs, magnetization is only slightly larger than the bulk value indicating that the surface effects are marginal in this case. From these results we infer that the noncompensation is the main factor for magnetization enhancement for thin $\mathrm{Fe}_{3} \mathrm{O}_{4}$ films. The orbital moment contribution from surface atoms also has to be ruled out as this is an effect comparable to the interface polarization effect. The uncompensated spin and extra charge associated with the surface might also lead to a much more profound change in the magnetic moment of the entire film. It may affect the antiferromagnetic exchange $\left(J_{A B}\right)$ between the $A$ - and $B$-layers underneath the surface, i.e., intersublattice exchange interaction. Slight decrease in the magnetization of 2 and $4 \mathrm{~nm}$ layers can be explained due to partial noncompensation which can arise either due to a small error in thickness of the film or roughness related effects. If we consider the noncompensation model along the growth direction and at the APBs, we can explain about 23\% enhancement for a 5-nm film. The estimate related to the APBs assumes: (a) domain size is $5 \mathrm{~nm} \times 5 \mathrm{~nm}$; (b) it is possible to overcome the AFM exchange by the application of magnetic field [18]; and (c) at the boundary stacking condition for the spinel structure, i.e., ABAB... is not satisfied.

\section{CONCLUSION}

From our study, we infer that the magnetite films, in particular at small thicknesses $(<5 \mathrm{~nm})$ are ferromagnetic and they possess magnetization values which are much larger than the values for bulk $\mathrm{Fe}_{3} \mathrm{O}_{4}$. We find that the noncompensation of spin moments between the two magnetic sub-lattices of the spinel
$\mathrm{Fe}_{3} \mathrm{O}_{4}$ along the growth direction and at the APBs is one of the major factors contributing to magnetization enhancement. Other possibilities such as influence of nonstoichiometry and noncompensation on the magnetic exchange interactions can not be completely ruled out.

\section{ACKNOWLEDGMENT}

The authors would like to gratefully acknowledge the financial support from the Science Foundation of Ireland (SFI) under Contract No. 06/IN.1/I91.

\section{REFERENCES}

[1] I. Zutic, J. Fabian, and S. Das Sarma, "Spintronics: Fundamentals and applications," Rev. Mod. Phys., vol. 76, no. 323, 2004.

[2] M. Ziese, Rep. Prog. Phys., vol. 65, no. 143, 2002.

[3] M. Fonin, Y. S. Dedkov, R. Pentcheva, U. Rudiger, and G. Guntherodt, "Magnetite: A search for the half-metallic state," J. Phys.: Condens. Matter, vol. 19, p. 315217, 2007

[4] D. T. Margulies, F. T. Parker, F. E. Spada, R. S. Goldman, J. Li, R. Sinclair, and A. E. Berkowitz, "Anomalous moment and anisotropy behavior in $\mathrm{Fe}_{3} \mathrm{O}_{4}$ films," Phys. Rev. B., vol. 53, p. 9175, 1996.

[5] W. Eerenstein, T. T. M. Palstra, S. S. Saxena, and T. Hibma, "Spinpolarized transport across sharp antiferromagnetic boundaries," Phys. Rev. Lett., vol. 88, p. 247204, 2002.

[6] S. Kale, S. M. Bhagat, S. E. Loafland, T. Scabarozi, S. B. Ogale, A. Orozco, S. R. Shinde, T. Venkatesan, B. Hannoyer, B. Mercey, and W. Prellier, "Film thickness and temperature dependence of the magnetic properties of pulsed-laser-deposited $\mathrm{Fe}_{3} \mathrm{O}_{4}$ films on different substrates," Phys. Rev. B., vol. 64, p. 205413, 2001.

[7] W. Eerenstein, T. T. M. Palstra, T. Hibma, and S. Celotto, "Origin of the increased resistivity in epitaxial $\mathrm{Fe}_{3} \mathrm{O}_{4}$ films," Phys. Rev. B., vol. 66, p. 201101, 2002.

[8] D. T. Margulies, F. T. Parker, M. L. Rudee, F. E. Spada, J. N. Chapman, P. R. Aitchison, and A. E. Berkowitz, "Origin of the anomalous magnetic behavior in single crystal $\mathrm{Fe}_{3} \mathrm{O}_{4}$ films," Phys. Rev. Lett., vol. 79 , p. 5162, 1997.

[9] F. C. Voogt, T. T. M. Palstra, L. Niesen, O. C. Rogojanu, M. A. James, and T. Hibma, "Superparamagnetic behavior of structural domains in epitaxial ultrathin magnetite films," Phys. Rev. B., vol. 57, p. R8107, 1998.

[10] S. K. Arora, R. G. S. Sofin, and I. V. Shvets, "Magnetoresistance enhancement in epitaxial magnetite films grown on vicinal substrates," Phys. Rev. B., vol. 72, p. 134404, 2005.

[11] Y. Zhou, C. McEvoy, R. Ramos, and I. V. Shvets, "The magnetic and magnetoresistance properties of ultrathin magnetite films grown on MgO substrate," J. Appl. Phys., vol. 99, p. 08J111, 2006.

[12] D. J. Huang, C. F. Chang, H. T. Jeng, G. Y. Guo, H. J. Lin, W. B. Wu H. C. Ku, A. Fujimori, Y. Takahashi, and C. T. Chen, "Spin and orbital magnetic moments of $\mathrm{Fe}_{3} \mathrm{O}_{4}$," Phys. Rev. Lett., vol. 93, p. 077204 2004.

[13] S. K. Arora, R. G. S. Sofin, I. V. Shvets, and M. Luysberg, "Anomalous strain relaxation behavior of $\mathrm{Fe}_{3} \mathrm{O}_{4} / \mathrm{MgO}(100)$ heteroepitaxial system grown using molecular beam epitaxy," J. Appl. Phys., vol. 100, p. 073908,2006

[14] G. Kresse and D. Joubert, "From ultrasoft pseudopotentials to the projector augmented-wave method," Phys. Rev. B., vol. 59, p. 1758, 1999.

[15] I. Leonov, A. N. Yaresko, V. N. Antonov, M. A. Korotin, and V. I. Anisimov, "Charge and orbital order in $\mathrm{Fe}_{3} \mathrm{O}_{4}$," Phys. Rev. Lett., vol. 93, p. 146404, 2004.

[16] H.-T. Jeng, G. Y. Guo, and D. J. Huang, "Charge orbital ordering and verwey transition in magnetite," Phys. Rev. Lett., vol. 93, p. 156403, 2004.

[17] Z. Lodziana, "Surface verwey transition in magnetite," Phys. Rev. Lett., vol. 99, p. 206402, 2007.

[18] T. Kasama, R. E. Dunin-Borkowski, and W. Eerenstein, Phys. Rev. B., vol. 73, p. 104432, 2006

[19] C. Cheng, "Structure and magnetic properties of $\mathrm{Fe}_{3} \mathrm{O}_{4}$ (001) surface: Ab-initio study," Phys. Rev. B., vol. 71, p. 052401.

Manuscript received March 03, 2008. Current version published December 17, 2008. Corresponding author: S. K. Arora (e-mail: aroras@tcd.ie). 